\title{
HEMORRAGIA DE TRONCO CEREBRAL APÓS REMOÇÃO CIRÚRGICA DE CISTO ARACNÓIDE DA FISSURA SILVIANA
}

\author{
RELATO DE CASO
}

GUILHERME BORGES*, YVENS B. FERNANDES**, NEVAIR R. GALLANI***

\begin{abstract}
RESUMO - Os autores relatam um caso de hemorragia de tronco cerebral após craniotomia para resseç̧ão de grande cisto aracnóide de fissura silviana esquerda. A sintomatologia inicial pré-operatória incluía sinais de hipertensão intracraniana e a tomografia computadorizada mostrava desvio de linha média. Diversos fatores são discutidos para explicar o sangramento parenquimatoso pós-operatório: edema cerebral, diminuição do retorno venoso e de fluxo sanguíneo no lado comprimido. Entretanto a fisiopatologia da hemorragia parenquimatosa em casos como o relatado permanece obscura. Uma abordagem cirúrgica mais cautelosa $\varepsilon$ proposta nesses pacientes com de hipertensão intracraniana.
\end{abstract}

PALAVRAS-CHAVE: cisto aracnóide, craniotomia, hemorragia do tronco cerebral, complicação cirúrgica.

Brainstem hemorrhage after surgical removal of arachnoid cyst of the sylvian fissure: case report

SUMMARY - The authors report a case of a hemorrhage of the brainstem after craniotomy for resection of a huge arachnoid cyst of the sylvian fissure on the left hemisphere. The previous simptomatology included clinical signs of increased intracranial pressure, and the computerized tomography showed midline shift. Some factors may contribute to brain hemorrhage post-operatively: cerebral edema, ipsilateral changes in the venous reflux and blood perfusion, although the physiopathology remains obscure. A more careful approach is suggested in such cases with intracranial hypertension.

KEY-WORDS: arachnoid cyst, craniotomy, brainstem hemornhage, surgical complication

O cisto aracnóide consiste de coleção de fluido recoberta por membrana similar à aracnóide, localizada entre a aracnóide e a piamater ${ }^{43}$. Ele representa $1 \%$ das lesōes expansivas intracranianas ${ }^{28}$. Leptomeningite cística, meningite crônica recidivante, aracnite adesiva circunscrita cística, meningite serosa e aracnopatia fibrocística proliferante são sinônimos encontrados na literatura com correspondência à entidade cisto aracnóide ${ }^{45}$. $\mathrm{O}$ cisto aracnóide congênito ou primário deve ser diferencidado de outras anormalidades congênitas ou adquiridas, tais como: cisto porencefálico, cisternas dilatadas, loculaçōes do espaço subaracnóide secundária a traumatismo craniano, hemorragia ou processo inflamatório ${ }^{3,10,13,15,29,33-36,44}$. Estes cistos heterogêneos sāo denominados cistos secundários $^{8}$. A origem do cisto aracnóide está provavelmente relacionada a desenvolvimento anormal da aracnóide, que pode se dividir ou duplicar ${ }^{83}$. O cisto aracnóide da fossa média é geralmente

*Disciplina de Neurocirurgia da Faculdade de Ciências Médicas da UNICAMP: "Professor Assistente Doutor; **Médico Residente; ***Pós-Graduando.

Dr Guilherme Borges - Rua Barão Geraldo de Rezende 282 - 13020-440 Campinas SP - Brasil. 
descoberto em pacientes com idade inferior a 20 anos; entretanto, pode ser encontrado em qualquer idade $^{8}$. É bem mais comum no sexo masculino e também mais frequente no lado esquerdo 9 . Sua sintomatologia é bastante diversa, incluindo cefaléia, epilepsia, hidrocefalia, hipertensão intracraniana, macrocefalia, proeminência da calvária (geralmente escama do temporal), paralisia de nervos cranianos, vertigem, proptose discreta, hemiparesia, retardo mental ou pode o mesmo vir a ser assintomático ${ }^{12,4,6,12,17,27}$. O quadro clínico em pacientes idosos é similar ao do hematoma subdural crônico ou hidrocefalia de pressão normal, geralmente sob a forma de demência, hemiparesia, ataxia, incontinência urinária ou dificuldade de deambulaçãa ${ }^{42}$.

Relatamos um caso de particular interesse quanto à conduta terapêutica.

\section{RELATO DE CASO}

AF, paciente do sexo masculino, 54 anos, procurou o serviço de Neurocirurgia da UNICAMP com queixa de cefaléia há 8 meses, diária, contínua, fronto-temporal esquerda (E) que cedia pouco com analgésicos comuns. Referia ainda episódios de parestesia acompanhada de discreta paresia em dimídio $\mathrm{E}$, de predomínio braquial. Relatava na ocasião que fora submetido a trépano-punção após ter sofrido queda no banheiro com consequente traumatismo craniano, há 2 anos. $O$ exame neurológico estava dentro dos limites da normalidade. Fundo de olho normal, embora sem pulso venoso espontâneo ou mesmo provocado. A tomografia computadorizada (TC) de crânio mostrava uma coleção na região do vale silviano à E causando desvio importante da linha média, não captante de contraste, compatível com cisto aracnóide (Fig 1).

Uma craniotomia fronto-temporal E com ressecção do cisto e comunicação ampla com as cisternas da base foi efetuada. A coloração do conteúdo líquido do cisto era ligeiramente xantocrômica. Não houve intercorrências durante o ato cirúrgico. No pós-operatório imediato evoluiu com hipertensão arterial, convulsões tônico-clônicas generalizadas de difícil controle e coma. A TC de crânio mostrava hematoma intracerebral à E (regiāo insular), hemorragia difusa en fossa posterior envolvendo o tronco cerebral e cerebelo, associado a

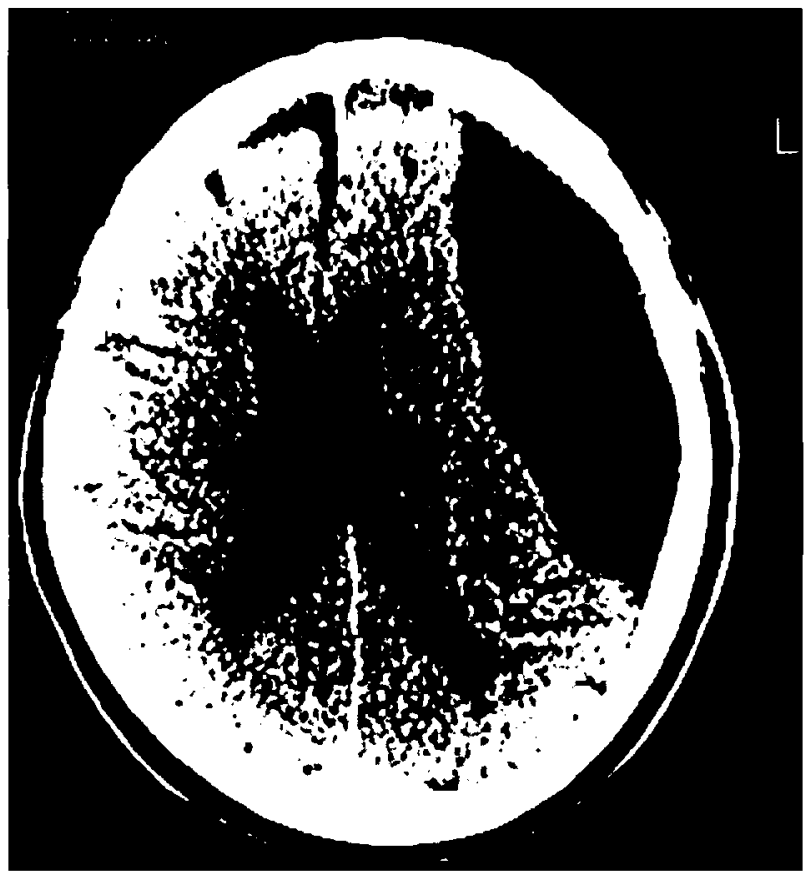

Fig 1. TC pré-operatório mostrando um grande cisto aracnóide em fissura silviana esquerda causando desvio da linha média. 


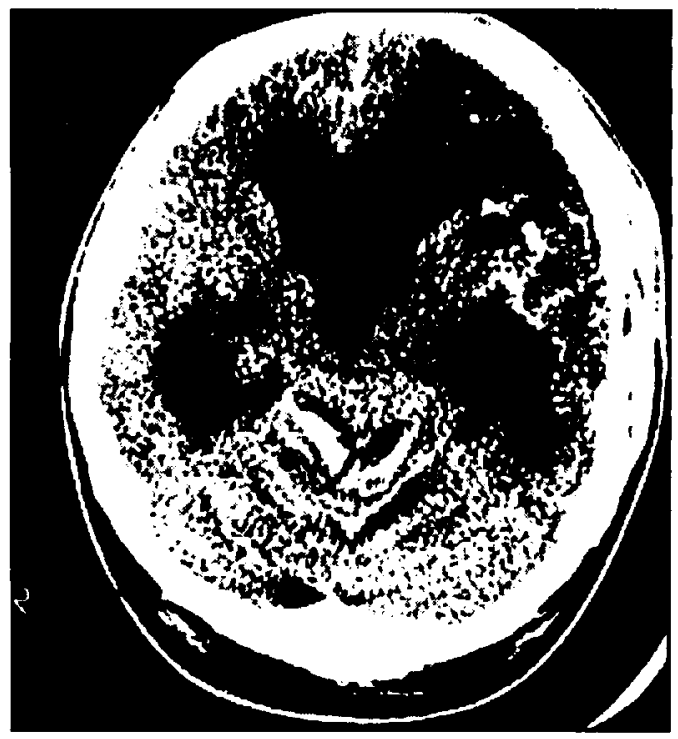

Fig 2. TC no pós-operatório imediato. Hemorragia difusa em fossa posterior e hidrocefalia aguda.

hidrocefalia aguda (Fig 2). Foi submetido imediatamente a derivação ventricular externa, falecendo, porém, uma semana após a cirurgia. O exame anátomo-patológico mostrou tratar-se de cisto aracnóide simples; entretanto havia grande quantidade de macrófagos com depósito de hemossiderina em sua cápsula (Fig 3).

\section{COMENTÁRIOS}

A terapêutica mais adequada para o cisto aracnóide ainda é assunto controverso ${ }^{1,2,6-}$ 8,19.21,24,32,34,41,42. Parece haver consenso em prol do tratamento conservador nos casos assintomáticos. Contudo, é sabido que nestes casos tratados conservadoramente existe maior risco de sangramento intracraniano (hematoma subdural ou hemorragia intra-cística) decorrente de traumatismo cranioencefálico, mesmo sendo este de leve intensidade ${ }^{18-21.26 .38}$. Possivelmente a origem do

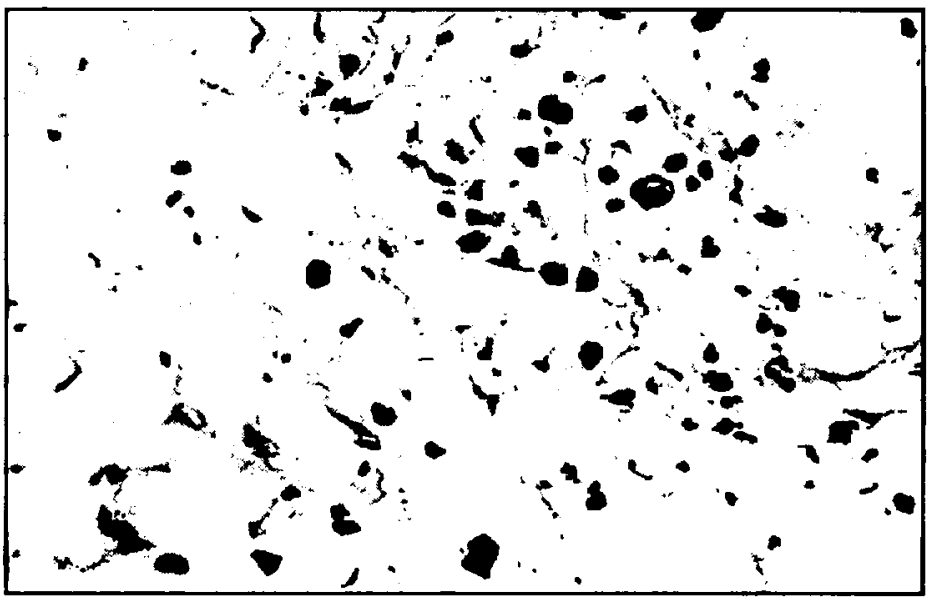

Fig 3. Cisto aracnóide. Nota-se a presença de macrófagos com depósito de hemossiderina. $H E, x 40$. 
sangramento estaria relacionada à ruptura de vasos delicados, tenuemente aderidos à cápsula do cisto $^{8}$. Isto teria levado alguns autores a indicarem a cirurgia nos casos assintomáticos. $\mathrm{O}$ tratamento cirúrgico baseia-se numa abordagem direta através de craniotomia com ressecção do cisto e comunicação com as cisternas da base, ou uma abordagem indireta através de derivação cistoperitoneal. A abordagem via craniotomia está associada a aproximadamente $25 \%$ de recorrência do cisto. As desvantagens da derivação são o mau funcionamento da válvula e o risco de infeç̧āo ${ }^{8}$. Săo descritos na literatura alguns casos de hemorragia ou mesmo óbito subsequente à cirurgia de cisto aracnóide, como também após a drenagem de hematoma subdural crônico ${ }^{1,5,11,22,23,25,37,39,40}$.

Klintworth 16 elegantemente demonstrou em estudo experimental que a rápida descompressão de lesões supratentoriais causa hemorragia no tronco cerebral e cerebelo, o que ele atribuiu ao fato de estarem essas estruturas deslocadas e sob isquemia, com diminuição do fluxo sanguíneo. A rápida descompressão levaria a grande aumento no fluxo sanguíneo, causando assim hemorragia.

McKissock $^{22}$ refere um caso de óbito secundário a hemorragia de tronco em um paciente operado de hematoma subdural crônico. Modesti e col. ${ }^{23}$ relatam 7 casos (5\% de sua casuística) de hematoma intracraniano após drenagem de coleções extracerebrais. A etiopatogenia desses sangramentos não está bem esclarecida. Diversos fatores poderiam contribuir para o seu surgimento: i) edema focal no tecido cerebral comprimido; ii) diminuição do retorno venoso no lado afetado; iii) inchaço subcortical na área comprimida pela lesão; iv) diminuição do fluxo sanguíneo regional no hemisfério ipsilateral, em casos de hematoma subdural crônico. Mais recentemente Spetzler e col. ${ }^{30}$ obtiveram evidências de perda da autorregulação e reatividade ao $\mathrm{CO} 2 \mathrm{em}$ hemisfério isquêmico de animais. A dilatação crônica de pequenos vasos arteriais, na presença de isquemia crônica, levaria a perda de sua autorregulação e resistência à pressão de perfusão normal. A descompressão cirúrgica causaria edema e hemorragias.

No presente caso o paciente tinha um grande cisto aracnóide de fissura silviana $\mathrm{E}$, com desvio importante da linha média. A indicação cirúrgica foi baseada na piora progressiva do quadro clínico nos últimos oito meses (sugestiva de hipertensão intracraniana). Havia também ausênsia de pulso venoso. Os exames pré-operatórios mostraram apenas discreta hiperglicemia não havendo qualquer alteração hematológica e nem hipertensão arterial sistêmica. $O$ exame anátomo-patológico foi importante para esclarecimento diagnóstico, porque habitualmente o cisto aracnóide é formado por células similares à aracnóide e nāo apresenta macrófagos com depósito de hemossiderina em sua cápsula $^{14}$. Isto explicaria, provavelmente, que o traumatismo craniano que o paciente sofrera dois anos antes, teria causado hemorragia intracística com posterior aumento do volume do cisto e, como consequência, teria provocado o agravamento do quadro clínico.

Em conclusão, a abordagem cirúrgica para o tratamento do cisto aracnóide é tecnicamente simples e aparentemente sem grandes complicações. Todavia, o caso apresentado vem confirmar que complicações graves, no tratamento cirúrgico de cistos grandes e com desvio importante das estruturas da linha média, carecem de estratégia especial. Embora a maioria dos autores indique abordagem direta, acreditamos que nestes casos uma primeira abordagem através de derivação cistoperitoneal permitiria descompressão mais lenta das estruturas intracranianas e consequente readaptação da microcirculação à pressão de perfusão circunstancial. A reavaliação do quadro clínico permitiria, na persistência da sintomatologia, utilizar abordagem direta contudo sempre, levando em consideração as graves complicações descritas.

Agradecimentos - Os autores agradecem ao Dr. Hugo L. F. Brito pelo material anátomo-patológico.

\section{REFERÉNCIAS}

1. Aicardi J, Bauman F. Supratentorial extracerebral cysts in infants and children. J Neurol Neurosurg Psychiatry 1975, 38:57-68.

2. Anderson FM, Hervey DS, Canton WL. Use of computerized tomography scanning in supratentorial arachnoid cysts: a report on 20 children and four adults. J Neurosurg 1979, 50:333-338. 
3. Bucy PC. Intrinsic tumors of the cerebellum and brainstem. In Bancroft FW, Pilchner C (eds). Surgical treatment of the nervous system. Philadelphia: J.B. Lipincott, 1946:212.

4. Choux M, Raybaud C, Pinsard N, Hassoun J, Gambarelli D. Intracranial supratentorial cysts in children excluding tumor and parasitic cysts. Child's Brain 1978, 4:15-32.

5. D'Avella D, De Blasi F, Rotilio A, Pensabene V, Pandolfo N. Intracerebral hematoma following evacuation of chronic subdural hematomas. J Neurosurg 1986, 65:710-712.

6. Dei-Anang, K. Klinik und Prognose der zerebralen Arachnoidalzysten, Inauguraldissertation, Johannes Gutenberg Universitaet. Mainz, 1982.

7. Dei-Anang K, Borges G, Schuermann K, Ludwig B. Cerebral arachnoid cysts. $20^{\circ}$ Congresso Latino Americano de Neurocirurgia, Sāo Paulo, Brasil, 19-24/março/83, Anais do Congresso:74-75.

8. Di Rocco C. Arachnoid cysts. In Youmans JR. Neurological surgery. Ed 3. Philadelphia: W.B. Saunders, 1990, Vol II: 1299-1325.

9. Galassi E, Piazza G, Gaist G, Frank F. Arachnoid cysts of the middle fossa: a clinical and radiological study of 25 cases treated surgically. Surg Neurol 1980, 14: 211-219.

10. Gardner WJ, Mc Cormack LJ, Dohn DF. Embryonal atresia of the fourth ventricle; the cause of arachnoid cysts of the cerebellopontine angle. J Neurosurg 1960, 17:226-237.

11. Go KG, Houthoff H, Blaauw E, Havinga P, Hartsuiker J. Arachnoid cysts of the sylvian fissure: evidence of fluid secretion. J Neurosurg 1984, 60:803-813.

12. Horrax G. Generalized cistemal arachnoiditis simulating cerebellar tumor: its surgical treatment and end results. Arch Surg 1924, 9:95-112.

13. Iglesias JR, Sanjuanbenito L, Martinez-Cubelis J, Lousa M, Redondo C. Intracerebral arachnoidal cyst containing choroid plexus: case report. Acta Neurochir (Wien) 1982, 58:229-234.

14. Jakubiak P, Dunsmore RH, Brecktett R. Supratentorial brain cysts. J Neurosurg 1968, 28: 129-136.

15. Jelsma F, Ross PJ. Traumatic intracranial arachnoidal cyst involving the gasserian ganglion: case report. J Neurosurg 1967, 26:439-441.

16. Klintworth GK. The pathogenesis of secondary brainstem hemorrhage as studied in an experimental model. Am J Pathol 1965, 47:525-536.

17. Little JR, Gomez MR, Mac Carthy CS. Infratentorial arachnoid cysts. J Neurosurg 1973, 39:380-386.

18. Lesoin F, Dhellemmes P, Rousseaux M, Jomin M. Arachnoid cysts and head injury. Acta Neurochir 1983, 69:43-51.

19. Markakis E, Heyer SL, Werry H. Die Aplasie der perisylvischen Region. Neurochirurgia 1980, 22:211-220.

20. Mayr U, Aichner F, Bauer G, Mohsenipour I, Pallua A. Supratentorial extracerebral cysts of the middle fossa: a report fo 23 consecutive cases of the so-called temporal lobe agenesia syndrome. Neurochirurgia 1982, 25:51-56.

21. Mc Cullough DC, Harbert JC, Manz HJ. Large arachnoid cyst at the cranial base. Neurosurgery 1980, 6:76-81.

22. McKissock W. Subdural hematoma: a review of 389 cases. Lancet 1960, 25:1365-1370.

23. Modesti L, Hodge CJ, Barmwell ML. Intracerebral hematoma after evacuation of chronic extracerebral fluid collections. Neurosurgery 1982, 10: 689-693.

24. Okonek G. Extracerebrale Arachnoidalzyste der linken Grosshimhemisphere. Zol Neurochir 1938, 3:112-119.

25. Övül KO. Intracerebral hematoma after evacuation of chronic subdural hematoma. Neurochirurgia 1938, 31:160-161.

26. Page A, Paxton RM, Mohan D. A reappraisal of the relationship between arachnoid cysts of the middle fossa and chronic subdural haematoma. J Neurol Neurosurg Psychiatry 1987, 50:1001-1007.

27. Robinson RG. Intracranial collections of fluid with local bulging of the skull. J Neurosurg 1955, 12:345-353.

28. Robinson RG. Congenital cysts of the brain: arachnoid malformation. Progr Neurol Surg 1971, 4:133-174.

29. Russel DS. Observations on the pathology of hydrocephalus. No. 256, 1-138, Her Majesty's Stationery Office. London, 1949-1952.

30. Spetezler RF, Wilson CB, Weinstein P, Mehdorn M, Townsend J, Telle D. Normal perfusion pressure breakthrough theory. Clin Neurosurg 1977, 25:651-672.

31. Starkman SP, Brown TC, Linell EA. Cerebral arachnoid cysts. J Neuropathol Exper Neurol 1958, 17:484-500.

32. Stein SC. Intracranial developmental cysts in children:treatment by cysto-peritoneal shunting Neurosurgery 1981, 8:647-650.

33. Taveras JM, Ransohoff J. Leptomeningeal cysts of the brain followin trauma with erosion of the skull: a study of seven cases treated by surgery. J Neurosurg 1953, 10:233-241.

34. Toennis W. Kongenitale Zysten der Zistemen. Zbl Neurochir 1937, 2:356.

35. Thompson RK. Cystic cerebellar arachnoiditis. J Neurosurg 1946, 3:461-467.

36. Trowbridge WV, French JD. Benign arachnoid cysts of the posterior fossa. J Neurosurg 1952, 9:398-404. 
37. Turtas S, Oranesu G. Intracerebral hematoma following removal of chronic subdural hematoma. Zbl Neurochir 1989, 50: 115-117.

38. Van der Meché FGA, Braakman R. Arachnoid cysts in the middle cranial fossa: cause and treatment of progressive and non-progressive symptoms. J Neurol Neurosurg Psychiatry 1983, 46:1102-1107.

39. Varma TRK, Sedzimir CB, Miles JB. Post-traumatic complications of arachnoid cysts and temporal lobe agenesis. J Neurol Neurosurg Psychiatry 1981, 44:29-34.

40. Williams B, Guthkelch AN. Why do central arachnoid pouches expand? J Neurol Neurosurg Psychiatry 1974, 37:1085-1092.

41. Wolpert SM, Scott RM. The value of metrizamidde CT cisternography in the management of cerebral arachnoid cysts. Am J Neurorad 1981, 2:29-35.

42. Yamakawa H, 'Ohkuma A, Hattori T, Niikawa S, Kobayashi H. Primary intracranial arachnoid cyst in the elderly: a survey on 39 cases. Acta Neurochir (Wien) 1991, 113:42-47

43. Zehnder M. Subarachnoidalsysten des Gehirns. Zbl Neurochir 1938, 3:100-112.

44. Zuelch KJ. Betrachtungen ueber die Entstehung der fruekindlichen Hirnschaedel auf Grund der klinischen und morphologischen Befunde. Arch Kinderheilk 1954, 149:1-27.

45. Zuelch KJ. Die Arachnitis ( A. adhaesiva cystica) In Handbuch der Neurochirurgie. Berlin SpringerVerlag, 1956, Band III: 603-615. 